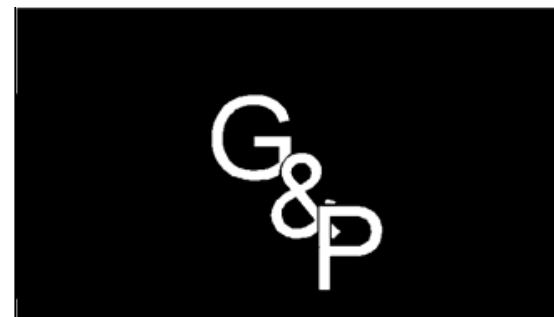

\title{
GESTÃO DO SISTEMA AGROINDUSTRIAL: A FORMAÇÃO DE RECURSOS HUMANOS PARA O AGRIBUSINESS BRASILEIRO.
}

\section{GESTÃO} $\&$

\section{PRODUÇÃO}

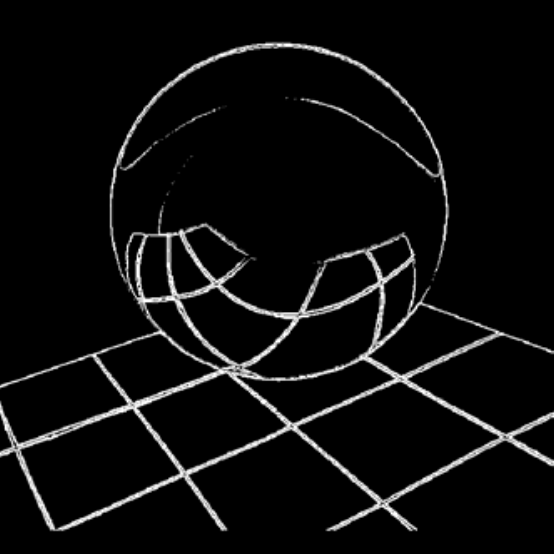

Prof. Dr. Mário Otávio Batalha

GEPAI - Grupo de Estudos e Pesquisas Agroindustriais DEP - Departamento de Engenharia de Produção UFSCar - Universidade Federal de São Carlos Via Washington Luís, km 235 - Caixa Postal 676 13565-905 - São Carlos - SP - Brasil Fone: (016) 2748236 - Fax: (016) 2748240

\section{Resumo}

O artigo inicia por uma breve apresentação do agribusiness brasileiro, sua importância para o país e os novos desafios que se apresentam para aumentar sua competitividade externa e a sua capacidade de abastecer o mercado interno de alimentos em quantidade $e$ qualidade adequadas. Nesta parte é salientada a importância vital da formação de profissionais voltados para as especificidades do sistema agroindustrial como forma de superar estes desafios. A seguir, o artigo identifica algumas iniciativas de instituições de ensino brasileiras que apontam nessa direção para, finalmente, apresentar a experiência pioneira da criação do curso de Engenharia de Produção Agroindustrial pela Universidade Federal de São Carlos. A estrutura curricular do referido curso é discutida e comenta-se a sua adaptação à tendência internacional de ensino em agribusiness.

\section{Palavras-chave: Agribusiness, Recursos Humanos, Agroindústria, Sistema Agroindustri- al, Engenharia de Produção Agroindustrial.}

\section{O Sistema Agroindustrial e a Economia Brasileira}

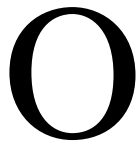

setor agroalimentar ocupa uma posição de destaque no sistema econômico de praticamente todos os países ditos desenvolvidos. Mesmo em países como a França, por exemplo, que possui um alto grau de competência tecnológica em várias áreas industriais, o sistema agroalimentar ainda representa o primeiro setor em valor de produção e um dos principais setores exportadores. Este é também o caso dos Estados Unidos (CONNOR, 1985). Na Alemanha e na Inglaterra, 
as indústrias agroalimentares (IAA) representam respectivamente $14 \%$ e $19 \%$ do valor da produção industrial (NEFUSSI, 1990). Mesmo o Japão, com o seu reduzido espaço territorial, representa atualmente a terceira potência agroindustrial do planeta (RASTOIN, 1992).

Estes dados não são mera coincidência. Eles evidenciam de forma inequívoca que uma economia forte e desenvolvida tem como uma das suas características o desenvolvimento harmonioso de todo o seu sistema agroindustrial. Além disso, esse desenvolvimento, nos países centrais, conta com uma forte proteção do setor primário, que se efetiva mediante expressivos investimentos do governo em infraestrutura, subsídios e barreiras (alfandegárias e não alfandegárias) à concorrência de produtos externos. Contar somente com uma agropecuária forte como forma de garantir o abastecimento interno e gerar divisas é um erro estratégico que o Brasil não pode cometer. O alimento deve ser produzido, industrializado e, finalmente, encaminhado até as mãos do consumidor. Qualquer disfunção em uma dessas etapas básicas compromete todo o esquema de abastecimento alimentar e de competitividade para o setor. Cabe acrescentar que esse alimento deve ainda ser produzido em padrões competitivos, que assegurem sua qualidade organoléptica, nutricional, bem como sua capacidade de atender às necessidades e peculiaridades do consumidor brasileiro.

Pode-se verificar que, também no Brasil, o sistema agroindustrial (SAI) é responsável por uma parte importante da economia nacional. Araújo et al. mostram que, em 1980, 46\% do orçamento familiar dos brasileiros estava comprometido com produtos relacionados ao SAI e que as atividades do SAI correspondiam a $32 \%$ do PIB brasileiro (ARAÚJO, 1989).

O sistema agroindustrial pode ser visto como um conjunto de seis grupos de atores econômicos diferentes: agricultura e pesca, indústrias agroalimentares, distribuição agrícola e alimentar, consumidor final, comércio internacional e indústria e serviços de apoio. Somente os agentes industriais deste conjunto representavam, em 1985, 20,8\% dos estabelecimentos industriais do país, empregando aproximadamente 13,3\% do total do pessoal ocupado (ABIA, 1994). Estes números tornam o sistema responsável pelo maior setor da indústria de transformação brasileira. Sua participação no Produto Interno Bruto se eleva a $11 \%$, praticamente o dobro, por exemplo, daquela da indústria automobilística.

As características estruturais da indústria agroalimentar lhe conferem algumas características que ajudaram, e tendem a continuar ajudando, a amortizar as bruscas variações que a economia brasileira vem sofrendo nos últimos anos. O aumento da população, aliado à baixa sensibilidade dos consumidores em relação ao consumo de grande parte dos gêneros alimentícios, faz com que o comportamento deste setor esteja menos sujeito a fortes oscilações de demanda. Em 1981, por exemplo, enquanto o PIB caía 4,4\% e a indústria de transformação em geral tinha queda de $10,2 \%$, a produção de alimentos agroindustrializados tinha um aumento de 2,7\% (ABIA, 1994).

Cabe mencionar que, ao contrário do que muitos pensam, 97,5\% do número total das agroindústrias nacionais são constituídas de micro e pequenas empresas, com até 99 empregados. É evidente que quando se analisa o valor da produção industrial esse número se altera substancialmente. Neste caso, as grandes e médias empresas são responsáveis por $63 \%$ do valor da produção industrial. Em relação ao número de empregados a situação praticamente se equilibra: 50,4\% dos empregos do setor são gerados pelas micro e pequenas empresas agroindustriais. Esta característica do parque agroindustrial torna-se relevante na medida em que são conhecidas as deficiências administrativas das P.M.E. nacionais, ao mesmo tempo que se reconhece sua existência como extremamente importante para o bom desempenho sócio-econômico do país (BATALHA, 1990). 
Apesar do enorme potencial do mercado agroalimentar brasileiro (em produção e em consumo) o Brasil ocupa uma posição relativamente modesta no comércio agroalimentar mundial. Em 1989 ele era responsável por 3\% do valor gerado pela agricultura mundial. Alguns autores estimam que esse valor sobe para 9\% se considerarmos somente os produtos agrícolas que já tenham sofrido uma primeira transformação industrial (JANK, 1990). No entanto, essa produção é suficiente para colocar o Brasil entre os principais produtores mundiais de vários produtos agrícolas (café, suco de laranja, cana de açúcar, banana, mandioca, soja, cacau, ...) e entre os oito primeiros países exportadores de alimentos.

A exportação de produtos agroalimentares continua sendo uma fonte importante de divisas para o pais. Em 1990 esses produtos foram responsáveis por 31\% do total das exportações brasileiras (JANK, 1990).

A importância estratégica do SAI para o Brasil pode ser visualizada segundo dois enfoques diferentes.

O primeiro deles é garantir um nível de abastecimento alimentar adequado à população brasileira. Para isso, não é suficiente somente uma agricultura forte mas é necessário também um setor agroindustrial eficiente e dinâmico. No Brasil, como em outras partes do mundo, existe uma tendência de consumo que se distancia cada vez mais dos produtos in natura para se aproximar dos produtos agroindustriais. $\mathrm{O}$ consumo de produtos alimentares industrializados no Brasil é da ordem de 49,31\%, havendo, portanto, um grande potencial a ser explorado para que seja alcançado o patamar dos países ditos desenvolvidos (ABIA, 1994). Estudos têm mostrado que processos agroindustriais adequados permitem diminuir o desperdício, regularizar os picos de produção e consumo e, ao contrário do que muitos pensam, oferecer produtos de maior qualidade para a população.

Aliado a este fator de abastecimento interno, $\mathrm{o}$ setor agroindustrial sempre desempenhou, e deve continuar desempenhando, um papel de destaque no equilíbrio do comércio exterior brasileiro.

No entanto, a moderna visão de agribusiness, que vem norteando o desenvolvimento do setor nas economias mais fortes do ocidente, coloca novos desafios para o setor agroindustrial brasileiro. A integração crescente da produção de insumos, da produção agropecuária, da agroindústria e da distribuição e armazenamento, bem como as constantes mudanças nos hábitos dos consumidores, tornam cada vez mais complexa a gestão das unidades de produção pertencentes ao SAI.

A situação no mercado externo, submetido a uma concorrência feroz, não é menos complexa. Ao mesmo tempo que a oferta se multiplica em vários dos mercados em que o Brasil mantém uma posição privilegiada, os países ditos desenvolvidos levantam barreiras à importação, como forma de preservar o seu mercado interno. De qualquer maneira, o Brasil não pode ficar alheio a essa tendência de internacionalização cada vez maior da economia mundial e de todas as conseqüências que ela acarreta.

A todos estes fatores, inerentes ao SAI, deve-se acrescentar a instabilidade econômica que vem assolando o país por um longo período, e que dificulta sobremaneira o gerenciamento das unidades produtivas nacionais.

É dentro desse contexto que a formação de recursos humanos competentes para abordar esta problemática resgata toda a sua importância. Vencer os desafios impostos pela necessidade de ser competitivo no âmbito internacional, não só pela exportação de commodities mas também, e principalmente, pela exportação de produtos com maior valor agregado, bem como garantir o abastecimento interno segundo as necessidades nutricionais e os anseios do consumidor brasileiro, exige, necessariamente, a formação de um corpo gerencial bem treinado e sintonizado com as peculiaridades da moderna visão de agribusiness. 


\section{O Ensino de Agribusiness no Brasil}

$\mathrm{O}$ despertar do meio empresarial internacional para as latentes vantagens competitivas do Brasil, tão logo lhe sejam dadas as condições de que desfrutam os países concorrentes, promoveu um alerta nos meios empresariais nacionais e um redirecionamento de prioridades nos meios políticos para a inegável vocação agroindustrial brasileira, e um sobressalto para as instituições de ensino e pesquisa que, por questões culturais e de orientação políticoestratégica, mantinham as atividades vinculadas à terra em segundo e terceiro plano. Desenvolver a agroindústria como forma de agregar valor aos produtos agropecuários gerados em suas regiões fez parte de praticamente todos os discursos políticos dos candidatos da campanha eleitoral de 1994.

O despertar do meio empresarial e político para a inegável vocação agroindustrial brasileira tem seus reflexos no meio acadêmico. Existem atualmente vários cursos que procuram formar profissionais com uma visão sistêmica que lhes permita atuar em um segmento específico do Sistema Agroindustrial sem, no entanto, perder de vista o funcionamento do conjunto. Nesse sentido, os cursos atuais de ensino em agribusiness no Brasil se coadunam com a tendência internacional.

Cabe ressaltar que, até o momento, grande parte desses cursos se concentra no nível de pós-graduação. O êxito dos primeiros cursos nessa área revelou uma demanda muito grande de profissionais desejando uma especialização voltada para a gestão dos negócios agroindustriais. Essa tendência foi claramente identificada por várias universidades e faculdades particulares, que passaram a oferecer cursos de especialização, muitas vezes, sem as devidas condições para tanto. Ao lado da iniciativa das escolas particulares, que oferecem cursos de especialização lato sensu existem várias universidades públicas que incluem no âmbito de seus programas de mestrado linhas de pesquisa destinadas a estudar e propor alternativas para o sistema agroindustrial. Cabe relembrar que estão sendo analisadas somente atividades relacionadas à gestão do sistema agroindustrial, estando excluídos os grupos de pesquisa que atuam na área de desenvolvimento de novos produtos, novos processos de produção, novas tecnologias, etc.

Também as escolas de agronomia, em face das dificuldades enfrentadas pelos agrônomos no mercado de trabalho, e cientes de que o papel desempenhado pela agricultura não pode mais ser dissociado daquele representado pelos outros agentes agregadores de valor das cadeias de produção agroindustriais, estão tentando cada vez mais orientar seus currículos para essa visão sistêmica que caracteriza a tendência atual de ensino, pesquisa e trabalho em agribusiness. Essa mudança encontra dificuldades oriundas da inércia dessas instituições, que, em alguns casos, carecem de profissionais formados dentro desta nova visão. O objetivo desta reorientação é expandir a área de atuação dos agrônomos, de forma a permitir uma maior e melhor compreensão do sistema no qual a agropecuária se insere, abrindo, desta forma, melhores perspectivas de emprego.

Recentemente, congressos importantes na área de Administração e Engenharia de Produção também estão abrindo um espaço valioso para a divulgação de artigos científicos vinculados à gestão e à regulação do Sistema Agroindustrial como um todo, bem como dos agentes econômicos que dele participam. Esse foi o caso dos Congressos da Associação Nacional de Pós-graduação em Administração, que iniciou, a partir de 1993, uma área temática intitulada "Administração Rural e Agroindustrial”. O ENEGEP (Encontro Nacional de Engenharia de Produção) também observa um incre- 
mento anual nas publicações relacionadas ao agribusiness. Os organizadores do XV ENEGEP e do Brazil First Congress of Industrial Engineering, reconhecendo essa importância crescente, estão prevendo uma mesa redonda, de caráter internacional, com o objetivo de discutir assuntos relacionados ao tema.

Ao lado dessas atividades de pósgraduação, vêm-se desenvolvendo outras iniciativas pioneiras no Brasil. A criação do curso de Engenharia de Produção Agroindustrial, na Universidade Federal de São
Carlos (UFSCar), representa uma dessas iniciativas. Este curso está no seu terceiro ano de existência e conta atualmente com noventa alunos. $\mathrm{O}$ aumento da relação candidato/vaga a cada ano reflete o êxito do curso. Cabe salientar que a criação do curso não passou desapercebida a outras entidades de ensino, que estão procurando o Departamento de Engenharia de Produção da UFSCar como forma de recolher subsídios para a implantação de cursos semelhantes em seus locais de origem.

\section{A Engenharia de Produção Agroindustrial}

$\mathrm{O}$ curso de Engenharia de Produção Agroindustrial da Universidade Federal de São Carlos, a exemplo dos cursos de pós-graduação citados anteriormente, foi criado como forma de suprir o mercado com profissionais especialmente adaptados à problemática que envolve os atores do Sistema Agroindustrial. Nesse sentido, o curso adota uma óptica sistêmica e procura formar profissionais capazes de atuar nos três grandes macrosegmentos que formam as cadeias de produção agroindustriais: agropecuária, industrialização e distribuição. Esta lógica pressupõe que um sistema de produção alimentar eficiente depende necessariamente da integração harmoniosa desses três segmentos produtivos. Um profissional que alie conhecimentos técnicos de produção peculiares a cada um desses segmentos e uma sólida formação em metodologias de gestão, além da indispensável compreensão das interfaces entre esses segmentos, possui atributos que lhe permitem vislumbrar excelentes oportunidades de colocação no meio acadêmico e empresarial.

A área privilegiada de atuação desses profissionais será, pelas características próprias dos cursos de Engenharia de Produção e que permeiam também o curso de Engenharia de Produção Agroindustrial, o setor agroindustrial. Mas, para tanto, algumas dificuldades devem ser superadas.
A grande maioria das ferramentas e metodologias clássicas de gestão industrial foi desenvolvida e vem sendo aplicada por indústrias outras que as ligadas ao sistema agroindustrial. Peculiaridades inerentes às agroindústrias (perecibilidade da matériaprima e do produto acabado, sazonalidade de consumo e de suprimento de matériasprimas, variação na qualidade da matériaprima, qualidade subjetiva do produto final, caráter político-social do abastecimento alimentar, etc) fazem com que grande parte dessas ferramentas tenha que ser adaptada para tornar-se aplicável a elas. Isso exige um considerável esforço de pesquisa, de âmbito nacional e internacional. Visando avançar nessa direção, o Departamento de Engenharia de Produção da UFSCar criou, em 1994, o GEPAI - Grupo de Estudos e Pesquisas Agroindustriais. Este grupo vem realizando pesquisas em várias áreas da gestão industrial, como forma de adaptar e estudar a aplicação das atuais ferramentas de gestão às necessidades específicas dos agentes econômicos do Sistema Agroindustrial. São áreas de atuação do GEPAI: Planejamento e Controle da Produção, Controle e Análise de Custos, Logística, Gestão da Qualidade, Marketing e Estratégia Industrial, Metodologias de Desenvolvimento de Novos Produtos, Simulação de Sistemas de Produção.

A implantação de cursos de graduação 
nos moldes do curso de Engenharia de Produção Agroindustrial esbarra na carência de profissionais com formação e experiência na área. A relativa falta de interesse da comunidade científica, que, durante anos, não soube avaliar a importância desta área de pesquisa e formação, não proporcionou a formação de quadros em quantidade suficiente para atender a atual demanda de profissionais. Nesse sentido, não existem dúvidas de que os centros de pesquisa e formação, atualmente em atividade, têm um papel importante na formação desses recursos humanos.

É importante salientar que, apesar dos esforços de divulgação empreendidos pelos pesquisadores e professores que atuam nessa área, o curso de Engenharia de Produção Agroindustrial ainda é relativamente desconhecido pela comunidade científica como um todo, e também pelo meio empresarial. Muitas vezes ele é confundido com cursos de Engenharia de Alimentos ou de Agronomia. Acredita-se que, com o ingresso das primeiras turmas no mercado de trabalho, aliado a um competente esforço de divulgação, será possível eliminar equívocos dessa natureza e ampliar as perspectivas de colocação para os profissionais com esse tipo de formação.

O currículo adotado procura espelhar a visão que norteou a criação do curso de Engenharia de Produção Agroindustrial, ou seja, uma abordagem sistêmica da problemática referente à oferta de produtos agroindustriais. Nesse sentido, sua análise permite identificar disciplinas relacionadas com os três macro-segmentos das cadeias de produção agroindustriais: produção agropecuária, industrialização e distribuição. Cabe salientar que estas disciplinas estão relacionadas aos conhecimentos técnicos de base de cada um dos segmentos, bem como abordam as ferramentas clássicas de gestão industrial utilizadas pela Engenharia de Produção.

A definicão inicial proposta por Goldberg considerava o agribusiness como sendo "o conjunto de todas as operações envolvidas na fabricação e distribuição de insumos e produtos agropecuários, bem como a armazenagem, processamento e distribuição de produtos agropecuários e produtos deles derivados” (GOLDBERG, 1974). A lógica da definição proposta por Goldberg dividia a cadeia de produção agroindustrial genérica em um macro-segmento produção de insumos, um macro-segmento central, representado pela produção de matériasprimas, e, finalmente, um macro-segmento processamento e distribuição. Os fundamentos que nortearam a criação do curso de Engenharia de Produção Agroindustrial adotam esta mesma óptica de agribusiness commodity system, porém com algumas modificações em relação à importância dada ao estudo de cada um dos macro-segmentos mencionados acima.

O programa curricular do curso admite implicitamente que, na grande maioria dos casos, é a dinâmica dos mercados ligados ao consumidor final dos produtos agroindustriais que dita, ao longo de toda a cadeia de produção agroindustrial, o ritmo das inovações tecnológicas e das condições concorrenciais às quais os agentes econômicos que participam da cadeia estão submetidos. As informações devem fluir do mercado para as agroindústrias, que devem adaptar seus produtos às novas exigências do consumidor. Em muitos casos essas adaptações demandam características próprias das matérias-primas existentes e novos processos de produção, o que faz com que esta nova demanda do consumidor tenha pronta repercussão nos setores de produção agropecuária e de insumos, bem como nas indústrias de apoio (eletrodomésticos, máquinas e equipamentos, máquinas agrícolas, etc). Esta constatação se coaduna com as modernas metodologias de análise industrial aplicadas ao SAI, que reconhecem no mercado o principal polo indutor de mudanças na dinâmica de funcionamento das cadeias agroindustriais e que, portanto, fazem dele o seu ponto inicial de análise, além de aspecto importante na delimitação dos contornos do espaço analítico a ser 
considerado (BATALHA, 1993).

Esta característica está contemplada no currículo adotado pelo curso, no qual foi previsto um conjunto de disciplinas que visa proporcionar ao profissional as ferramentas necessárias à identificação, junto ao mercado, das tendências de consumo e das modificações estruturais e conjunturais que podem modificar essas tendências. Fazem parte desse conjunto de disciplinas: Sistemas Agroindustriais, Marketing Estratégico Agroindustrial, Comportamento do Consumidor e Pesquisa de Mercado e Comercialização dos Produtos Agroindustriais.

As disciplinas básicas de gestão industrial estão sendo adaptadas às especificidades das agroindústrias para proporcionar conhecimentos que permitam o gerenciamento eficaz e eficiente das unidades industriais que participam ao processo. Fazem parte desse conjunto de disciplinas: Sistemas de Informação, Gestão da Qualidade, Custos Agroindustriais, Planejamento e Controle da Produção, Contabilidade e Finanças, etc. Os conhecimentos tecnológicos imprescindíveis à boa formação profissional do estudante estão garantidos pela inclusão disciplinas que envolvem Processos Químicos Agroindustriais e Operações Unitárias Agroindustriais.

No que tange aos conhecimentos específicos ligados ao setor primário de produção, estão previstas disciplinas que envolvem conhecimentos básicos em produção agropecuária (Fatores de Produção Agropecuária), Gerenciamento da Propriedade Rural, Economia Agrícola, Relações de Trabalho no Campo e Organização da Agricultura Brasileira e Mundial.

Esse conjunto de conhecimentos específicos, aliado às disciplinas tradicionais que compõem o escopo dos cursos de Engenharia de Produção no Brasil, permite a formação de um profissional diferenciado em relação aos egressos dos cursos existentes. A presença desses profissionais no mercado de trabalho proporcionará às instituições públicas e privadas, recursos humanos treinados e sintonizados com as peculiaridades do Sistema Agroindustrial, o que, sem nenhuma dúvida, contribuirá para superar os desafios impostos ao agribusiness brasileiro.

Finalmente, cabe destacar que a preocupação com a formação de recursos humanos destinados ao agribusiness existe também em vários outros países. Nos Estados Unidos, por exemplo, uma pesquisa realizada pela Texas A \& $M$ University junto a 543 firmas ligadas ao sistema agroindustrial revelou as principais competências gerenciais que aquelas empresas buscam encontrar nos seus profissionais. Um resultado parcial da pesquisa pode ser visto na Tabela 1. Convém destacar que o conjunto de conhecimentos apresentados nessa tabela encontra-se, de maneira mais ou menos enfática, representado no currículo do curso de Engenharia de Produção Agroindustrial.

Além desses aspectos, a pesquisa apontou como característica importante para os profissionais do agribusiness a "capacidade de comunicação". Tais características seriam: capacidade de dar instruções claras e concisas, expressar claramente suas idéias (por escrito e oralmente), redação de relatórios claros e concisos, etc. Assim, também é importante que as escolas formadoras de recursos humanos para o agribusiness não negligenciem esses aspectos. Essas habilidades podem ser estimuladas e ensinadas no bojo de todas as disciplinas que compõem o currículo do curso. Uma maneira interessante de alcançar esse objetivo é estimular o uso de estudos de caso nas disciplinas. Embora suscite controvérsias à sua eficacidade, o estudo de casos presta-se muito bem a exercícios desse tipo. A obrigatoriedade da apresentação, por parte dos estudantes, de relatórios e exposições orais sobre os casos estudados pode contribuir grandemente para que se alcancem tais objetivos. 


\section{Conclusão}

$1 \begin{gathered}\text { agropecuária brasileira ocupa } \\ \text { atualmente uma posição privilegia- } \\ \text { da dentro do cenário mundial. O }\end{gathered}$ país está entre os maiores produtores e exportadores de alimentos do planeta.
No entanto, é imprescindível que o agribusiness brasileiro saiba reconhecer as novas tendências internacionais para o setor, ao mesmo tempo que procura acompanhá-las.

\section{Tabela 1 - Ranking dos Mais Importantes Conhecimentos em Gestão e Economia para Profissionais do Agribusiness. \\ Fonte: LOVE (1992)}

\begin{tabular}{|l|c|c|}
\hline \multicolumn{1}{|c|}{ Características } & Posição & Nota média \\
\hline Identificação de metas e objetivos para a empresa & 1 & 7,840 \\
\hline Identificação das áreas críticas para a empresa & 2 & 7,821 \\
\hline Coordenação de recursos humanos e materiais & 3 & 7,698 \\
\hline Desenvolvimento de estratégias empresariais & 4 & 7,326 \\
\hline Capacidade de leitura e utilização de registros financeiros & 5 & 7,254 \\
\hline Marketing & 6 & 7,060 \\
\hline Identificação e administração de riscos & 7 & 7,026 \\
\hline Conhecimentos em vendas & 8 & 6,882 \\
\hline Planejamento de Recursos Humanos & 9 & 6,810 \\
\hline Microeconomia & 10 & 6,785 \\
\hline Utilização de conceitos de custos & 11 & 6,657 \\
\hline Conhecimentos de finanças de empresas & 12 & 6,361 \\
\hline Política agrícola dos EUA & 13 & 5,910 \\
\hline Sistemas de administração de materiais & 14 & 5,783 \\
\hline Estrutura organizacional & 15 & 5,733 \\
\hline Estudos de leiaute & 16 & 5,634 \\
\hline Macroeconomia & 17 & 5,611 \\
\hline Política nacional e internacional & 18 & 5,538 \\
\hline Comércio internacional & 19 & 5,037 \\
\hline Economia internacional & 20 & 4,634 \\
\hline
\end{tabular}

Além disso, é preciso ultrapassar o modelo exportador de commodities agrícolas, que por muitos anos caracterizou as exportações do país. É vital que essas commodities sofram transformações industriais no Brasil, de forma a agregar valor a uma matériaprima escassa e, assim, gerar mais riquezas em solo nacional.

Cabe ainda salientar que a grande produção agropecuária brasileira não foi capaz de eliminar o grave problema da fome, que ainda assola regiões inteiras do país, não em decorrência de falta de alimentos mas sim da falta de condições para adquiri-los. Não existem dúvidas de que o incremento da produção de produtos alimentares industrializados, bem como uma gestão melhor de todo o sistema de produção agroindustrial, pode contribuir substancialmente para a resolução desse problema. É sabido que cadeias de produção agroindustriais bem equilibradas diminuem desperdícios, aumen- 
tam a qualidade dos produtos e permitem eliminar sazonalidades de oferta e demanda, atuando, dessa forma, no próprio mecanismo de formação de preços dos alimentos, possibilitando uma economia mais estável.

Porém, para explorar as vantagens competitivas do Brasil na área agroindustrial, torna-se imperativo que as empresas e o poder público possam contar com profissionais capacitados e familiarizados com a problemática que envolve o funcionamento do sistema agroindustrial. De certa forma, este fato já vem sendo reconhecido pelas empresas privadas e pelo setor público que, nos últimos anos, têm multiplicado iniciativas neste sentido. É dentro deste contexto que a implementação de cursos como o de Engenharia de Produção Agroindustrial assume uma grande importância.

\section{Referências Bibliográficas:}

ABIA: Programa brasileiro de qualidade e produtividade: subprograma setorial do sistema agroindustrial. Termo de referência. ABIA, São Paulo, 1994.

ARAÚJO, N. BITTENCOURT DE et al.: O complexo agroindustrial brasileiro. Agroceres, São Paulo, 1989.

BATALHA, M. O.: La notion de filière comme outil d'analyse stratégique: le cas des matières grasses à tartiner au Brésil.. Tese de doutorado, INPL, Nancy, França. 1993.

CONNOR, J.: The food manufacturing industries.. Lexington Books,, Massuchusets, EUA, 1985.

GOLDBERG, R. A.: Agribusiness management for developing countries - Latin America. Ballinger Publishing Company, Cambridge, USA, 1974.
JANK, S.: A inserção do Brasil e do setor cooperativo no mercado mundial de produtos agroindustrializados. USP/ESALQ, Piracicaba - São Paulo, 1990.

LOVE, H. G.: Designing and using case studies in agribusiness education. Seminário apresentado na Escola Superior de Agricultura Luiz de Queiroz, Universidade de São Paulo, Piracicaba, São Paulo, em 26 de agosto de 1992.

NEFUSSI, J.: Les industries agroalimentaires. Collection que sais-je? PUF, Paris, França, 1990.

RASTOIN, J. L.: "Les multinationales et le système alimentaire mondial: tendances stratégiques”. In: Economies et Sociétés, Série Développement agroalimentaire, AG 21, Paris, França, 1992.

\section{Bibliografia Complementar:}

ALEXANDRATOS, N.: L'agriculture mondiale: horizon 2000. Collection économie agricole \& agro-alimentaire. Economica. Paris, França, 1989.

BATALHA, M. O. \& FLORIOT, J. L.: “A new method for strategic analysis in agribusiness: the case of spreadble fats in Brazil". In: Symposium de l'International Agribusiness Management Association, San Francisco, USA, maio/1993.

BATALHA, M. O. \& STHALBERG, P.: "A gestão da produção em firmas agroindustriais”. In: Anais do XIV ENEGEP, João Pessoa - PB, pp. 42-47, 1994.
BATALHA, M. O. \& TOLEDO, J. C.: "A gestão da qualidade no sistema agroindustrial”. In: Anais do XIV ENEGEP, João Pessoa - PB, pp. 1154a-1154f, 1994.

BATALHA, M. O.: A pequena e média indústria em Santa Catarina. Ed. da UFSC, Florianópolis, SC, 1990

CASWELL, J. A.: "Using industrial organization and demand models for agribusiness research”. Agribusiness, John Wiley \& Sons, Vol. 8, n 6, pp. 537-548, 1992.

CHARVET, J. P.: La désorde alimentaire mondial. Hatier, Paris, França, 1987.

CONNOR, J. M.: Food Processing: an industrial powerhouse in transition. Lexington Books, Massachusetts, USA, 1988. 
FARINA, E.M.M.Q. \& ZYLBERSJTAJN,D.: Competitividade e organização nas cadeias agroindustriais. Texto para discussão. FEA/USP, 1994.

FLORIOT, J. L.: "Les stratégies industrielles et la maîtrise des flux en industries agroalimentaires". Revue Economie \& Gestion Agro-alimentaire, $\mathrm{n}^{\circ}$ 24, Cergy-Pontoise, França, julho/1992.

MALASSIS, L.: "L'économie agroalimentaire: une discipline en développement". In: Economie Agroalimentaire: concepts et méthodes. Cahiers de l'ISMEA, AG n²1, 1992.

MALASSIS, L.: Economie Agro-alimentaire. Cujas, Paris, França, 1979.
MARION, B. W.: The organization and performance of the US food system. Lexington Books, Massachusetts, USA, 1986.

RAMA, R.: Investir dans l'industrie agroalimentaire. OCDE, Paris, França, 1992.

TRUZZI, O. M. S.; COSTA, M. A. B. \& MORABITO, R.: "Engenharia de produção agroindustrial: um novo curso de graduação”. In: Anais do XIII ENEGEP, Florianópolis - SC, 1993.

\section{AGRI-FOOD MANAGEMENT: DEVELOPING HUMAN RESOURCES FOR THE BRAZILIAN AGRIBUSINESS.}

\section{Abstract}

The article provides a general overview of Brazilian agribusiness and the new challenges for improving its external competitivity as well as for supplying the internal food market adequately in terms of quantity and quality. The crucial importance of the education of professionals in agrifoods management as a condition to the development of Brazilian agribusiness is highlighted. The article identifies how Brazilian Universities are concretely working towards this goal. Finally, the course of Agri-food Industrial Engineering at the Federal University of São Carlos is examined regarding its adaptation to international trends in agribusiness education. 